\title{
Stimulation of the noradrenergic system enhances and blockade reduces memory for emotional material in man
}

\author{
R. E. O'CARROLL, ${ }^{1}$ E. DRYSDALE, L. CAHILL, P. SHAJAHAN AND K. P. EBMEIER \\ From the Department of Psychology, University of Stirling, Stirling and MRC Brain Metabolism Unit, Royal \\ Edinburgh Hospital, Edinburgh; and Centre for Neurobiology of Learning and Memory, Department of \\ Psychobiology, University of California, Irvine, CA, USA
}

\begin{abstract}
Background. It is clearly established that emotional events tend to be remembered particularly vividly. The neurobiological substrates of this phenomenon are poorly understood. Recently, the noradrenergic system has been implicated in that beta blockade has been shown to reduce significantly the delayed recall of emotional material with matched neutral material being unaffected.

Methods. In the present study, 36 healthy young adults were randomly allocated to receive either yohimbine, which stimulates central noradrenergic activity, metoprolol which blocks noradrenergic activity, or matched placebo. The three groups were well matched. All capsules were taken orally, prior to viewing a narrated 11 slide show described a boy being involved in an accident.

Results. Yohimbine significantly elevated, and metoprolol reduced mean heart rate during the slide show relative to placebo, thus confirming the efficacy of the pharmacological manipulation. One week later, in a 'surprise' test, memory for the slide show was tested. As predicted, yohimbinetreated subjects recalled significantly more and metoprolol subjects fewer slides relative to placebo. This result was confirmed via analysis of multiple-choice recognition memory scores.
\end{abstract}

Conclusions. We conclude that stimulation of the noradrenergic system results in the enhancement and blockade in a reduction of recall and recognition of emotional material in man.

\section{INTRODUCTION}

It is clearly established that emotional events tend to be remembered particularly vividly (Christianson, 1992). Over a century ago, William James stated 'An experience may be so exciting emotionally as almost to leave a scar on the cerebral tissues' (James, 1884). A recent demonstration of this phenomenon was reported by Ikeda et al. (1998), who studied 51 Alzheimer patients who had experienced the Kobe earthquake in Japan and were studied 6 to 10 weeks after the disaster. All had received an MRI brain scan in the intervening period. It was hypothe-

${ }^{1}$ Address for correspondence: Professor R. E. O'Carroll, School of Psychology, University of St. Andrews, St. Andrews, Fife KY16 9JU. sized that both events would be highly memorable, but that the earthquake would have evoked significantly more emotion than the MRI. The earthquake was remembered by $86.3 \%$ of the subjects, however, only $31.4 \%$ remembered having the MRI scan. This study is an example of the phenomena whereby emotion reinforces memory for an event, even in subjects with compromised memories.

In recent years, there has been a renaissance in the study of emotion in cognitive neuroscience (LeDoux, 1996; Cahill \& McGaugh, 1998). While it has been well recognized that emotional experiences are often remembered particularly vividly, the underlying neurobiological mechanisms responsible for this phenomenon have been poorly understood. Cahill et al. (1994) 
demonstrated that beta-adrenergic activity was involved in memory for emotive material in humans. Healthy young adults were shown a series of slides with either a neutral or arousing accompanying narrative. In the central section of the arousing slide show, a boy has a serious car crash, is nearly killed, and has to have his feet surgically reattached. Half the subjects received a single $40 \mathrm{mg}$ capsule of the beta blocker propranolol, $1 \mathrm{~h}$ before the slide presentation and the other half received placebo. One week later in a 'surprise' memory test, Cahill et al. (1994) showed that the beta blockade had selectively impaired recall and recognition for the emotive central story section in the propranolol-treated subjects. Further studies have implicated the amygdala in this system (Cahill et al. 1995, 1996). Subsequent attempts to replicate the Cahill et al. (1994) beta blockade study have produced conflicting results (van Stegeren et al. 1998; O'Carroll et al. 1999). The present study attempted to test the hypothesis that stimulation of the central noradrenergic system would result in an enhancement, and blockade in a reduction, of memory for emotional material in man.

\section{METHOD}

Thirty-six healthy young adults were recruited from local universities. They were told that we were interested in their physiological and subjective responses to arousing material. All subjects gave informed consent and the study was approved by the University and local Health Service NHS Trust Ethics Committees. Prospective subjects were told that they would participate in the experiment which would consist of two sessions, 1 week apart, and they were asked not to discuss the study with anyone in the intervening week. Subjects were randomly allocated to one of three conditions: (a) $20 \mathrm{mg}$ yohimbine hydrochloride, which stimulates central noradrenergic activity via blockade of the alpha-2 adrenergic autoreceptor (Charney et al. 1987; Peskind et al. 1995); (b) 50 mg metoprolol, a widely used clinical beta blocker, (selective to the beta-1 receptor (Walker, 1991)); or (c) matched placebo. The subjects were all assessed for the potential confounding variables of age, sex, intelligence (Nelson \& Willison, 1991) general memory functioning (Delis et al. 1987), extroversion and neuroticism (Eysenck et al. 1985), basal blood pressure and pulse rate.

\section{Procedure}

All capsules were taken orally $90 \mathrm{~min}$ prior to viewing a narrated 11 slide show in a doubleblind design. This time was selected so as to allow for peak plasma drug levels at time of stimulus exposure (Peskind et al. 1995; Walker, 1991). In this experiment, only the emotionally arousing narrative was used. The slides and story describe a boy being involved in an accident with the emotional story elements being introduced in the central phase of the slide show (Cahill et al. 1994; Cahill \& McGaugh, 1995). All subjects had continuous heart monitoring throughout the narrated slide show using the Biopac MP30 system. Immediately following the slide show, subjects rated their personal emotional reactions to the slides, using an 0-10 scale, following Cahill et al. (1994). One week following the initial phase of the study, subjects returned to the laboratory and were told that, in fact, the focus of the study was memory for the material that was presented the previous week. They were asked to recall freely as much as they could of the slides and story. Thereafter, they were told that they, in fact, had seen 11 slides and were asked to describe as many of the slides as they could remember seeing. They were then given a multiple choice test regarding their detailed memory for each slide. Five to nine questions per slide were asked, following Cahill et al. (1994), for example: 'Whose picture is in slide 1?' (a) mother and her son; (b) father and his son; $(c)$ mother and father; $(d)$ no one is pictured. Scoring was conducted with the experimenter blind to drug condition. All subjects were asked whether they had guessed the memory would be assessed at the second session, and none indicated that they had. Full details of the methodology are provided by in Cahill et al. (1995) and Cahill \& McGaugh (1995).

\section{Statistical analysis}

Between-group differences in matching variables were tested for using ANOVA and chi-square statistics. For the main experimental hypothesis, we predicted an ordered effect, namely that yohimbine would stimulate noradrenergic function and metoprolol would block noradrenergic function, relative to placebo. We, therefore, 
predicted an ordered effect of yohimbine $>$ placebo $>$ metoprolol and accordingly employed the Jonckheere non-parametric trend test as the principal test of our experimental hypothesis (Coolican, 1994), thus obviating the need for post hoc comparisons.

\section{RESULTS}

No significant differences emerged between the three subject groups in terms of any of potential confounding variables (see Table 1). There was no significant difference between the groups in mean (S.D.) number of days elapsed between slide presentation and memory test (metoprolol $6.4(0.9)$ days, yohimbine 6.7 (1.9) days and placebo $6.6(1.2)$ days, $F=0.93, P=0.91$. Yohimbine significantly elevated and metoprolol reduced mean heart rate during the slide show, relative to placebo, thus confirming the efficacy of the pharmacological manipulation (metoprolol $=53.8(8 \cdot 1)$, placebo $=63.8(11.8)$, yohimbine $=71.6(10.8)$, Jonckheere $Z=3.85, P<$ $0 \cdot 0001)$. Immediately following the slide presentation, there was no significant between-group difference in subjective emotional reaction to the story and slides recorded using a 0-10 scale, following Cahill et al. (1994): metoprolol rating mean $=3 \cdot 8$, standard deviation $=2 \cdot 1$; placebo $=4.7(1.9), \quad$ yohimbine $=4.5(2.8), \quad F=0.53$, $P=0.59$.

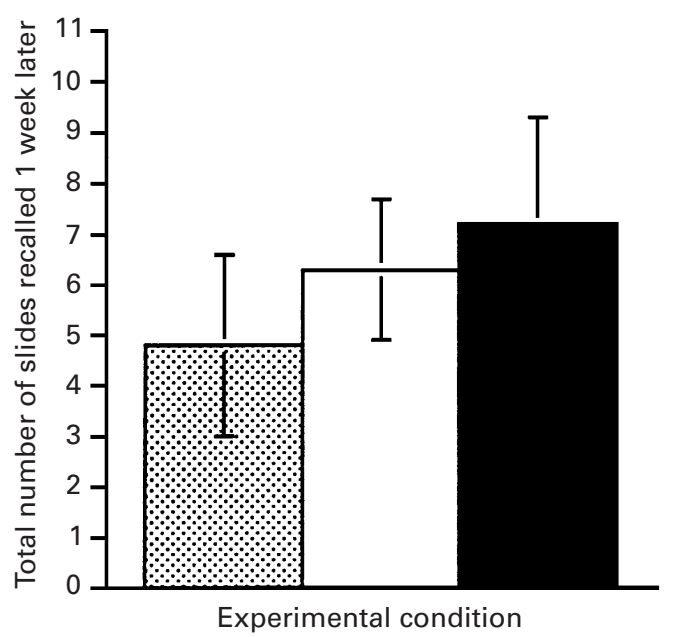

Fig. 1. Total number of slides recalled: predicted order effect metoprolol ( 0 ) impairs and yohimbine ( $\square$ ) improves memory relative to placebo $(\square)$. (Jonckheere trend test, $Z=2.39 ; P<0.01$.)

Seven days later, memory for the slides and story was tested. Subjects were credited with recall of a slide if they described some information that could only have been known from viewing that particular slide. As predicted, yohimbine subjects recalled significantly more and metoprolol fewer total number of slides relative to placebo (see Fig. 1). The 11 slide story can be broken into three phases, slides 1-4 (phase 1) introduce the story and are relatively neutral, slides 5-8 (phase 2) feature the accident,

Table 1. Three groups matched on potential confounding variables

\begin{tabular}{|c|c|c|c|c|c|}
\hline & $\begin{array}{c}\text { Metoprolol } \\
\quad N=12 \\
\text { Mean (S.D.) }\end{array}$ & $\begin{array}{c}\text { Yohimbine } \\
\quad N=12 \\
\text { Mean (s.D.) }\end{array}$ & $\begin{array}{c}\text { Placebo } \\
N=12 \\
\text { Mean (S.D.) }\end{array}$ & $F$ & $P$ \\
\hline Age & $21 \cdot 1(2 \cdot 7)$ & $23 \cdot 2(7 \cdot 5)$ & $21 \cdot 3(2 \cdot 2)$ & 0.698 & 0.505 \\
\hline CVLT (1-5) & $59 \cdot 1(7 \cdot 0)$ & $58.4(7 \cdot 8)$ & $58 \cdot 1(10 \cdot 1)$ & 0.044 & 0.957 \\
\hline CVLT del & $13 \cdot 8(2 \cdot 7)$ & $13 \cdot 2(2 \cdot 5)$ & $13 \cdot 4(2 \cdot 2)$ & $0 \cdot 218$ & $0 \cdot 806$ \\
\hline CVLT disc & $97 \cdot 8(3 \cdot 2)$ & $97 \cdot 3(3 \cdot 6)$ & $98 \cdot 1(2 \cdot 8)$ & $0 \cdot 213$ & $0 \cdot 809$ \\
\hline NART IQ & $111.6(7.6)$ & $110 \cdot 8(7 \cdot 8)$ & $110 \cdot 7(8 \cdot 7)$ & $0 \cdot 043$ & 0.958 \\
\hline $\mathrm{N}$ & $12 \cdot 0(6 \cdot 0)$ & $10 \cdot 7(5 \cdot 9)$ & $7 \cdot 8(3.6)$ & 1.939 & $0 \cdot 159$ \\
\hline $\mathrm{E}$ & $17 \cdot 5(2 \cdot 4)$ & $16 \cdot 0(4 \cdot 7)$ & $16 \cdot 1(2 \cdot 8)$ & $0 \cdot 722$ & 0.493 \\
\hline $\mathrm{P}$ & $4 \cdot 4(3 \cdot 3)$ & $3 \cdot 8(1.9)$ & $3 \cdot 5(2 \cdot 1)$ & $0 \cdot 411$ & 0.667 \\
\hline $\mathrm{L}$ & $4.0(1.8)$ & $5 \cdot 8(2 \cdot 9)$ & $6 \cdot 5(2 \cdot 5)$ & $3 \cdot 409$ & $0 \cdot 045$ \\
\hline Pre Sys & $121 \cdot 6(13 \cdot 7)$ & $125 \cdot 3(9 \cdot 7)$ & $121 \cdot 8(9 \cdot 9)$ & $0 \cdot 426$ & 0.657 \\
\hline Pre Dias & $67 \cdot 8(10 \cdot 7)$ & $72 \cdot 3(9 \cdot 2)$ & $70 \cdot 9(4 \cdot 8)$ & $0 \cdot 864$ & $0 \cdot 431$ \\
\hline Pre Pulse & $68 \cdot 3(14 \cdot 3)$ & $74 \cdot 3(9 \cdot 3)$ & $68 \cdot 3(12 \cdot 4)$ & $0 \cdot 959$ & $0 \cdot 394$ \\
\hline Sex & $6 \mathrm{M}, 6 \mathrm{~F}$ & $7 \mathrm{M}, 5 \mathrm{~F}$ & $5 \mathrm{M}, 7 \mathrm{~F}$ & $\chi^{2}=0.67$ & 0.716 \\
\hline
\end{tabular}

Key: CVLT (1-5), California Verbal Learning Test List A Trials 1-5 raw score; CVLT del, California Verbal Learning Test delayed free recall List A; CVLT disc, California Verbal Learning Test recognition discrimination score;' NART IQ, National Adult Reading Test predicted pre-morbid full scale IQ; E, Extroversion score from Eysenck Personality Questionnaire-Revised; N, Neuroticism score from Eysenck Personality Questionnaire-Revised; P, Psychoticism score from Eysenck Personality Questionnaire-Revised; L, Lie score from Eysenck Personality Questionnaire-Revised; Pre Sys, baseline systolic blood pressure; Pre Dias, baseline diastolic blood pressure; Pre Pulse, baseline pulse rate. 


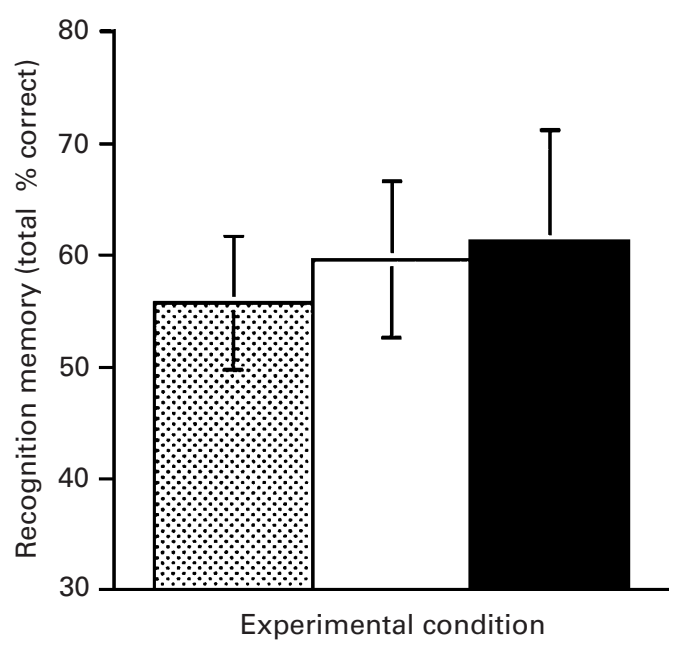

FIG. 2. Recognition memory: metoprolol () impairs and yohimbine ( $\square$ ) improves memory relative to placebo $(\square)$. (Jonckheere trend test, $Z=1.90 ; P<0 \cdot 05$.)

where a boy gets knocked down and surgeons then reattach his severed feet and are emotionally arousing, slides 9-11 (phase 3) describe the mother leaving the hospital to pick up her other child. Previous work has demonstrated that healthy subjects typically demonstrate heightened recall for phase 2. Accordingly, breaking down the number of slides recalled by story phase revealed a significant predicted ordered effect restricted to the central four slide emotional phase of the story (Phase 1 metoprolol= $1.7(0.9), \quad$ placebo $=2 \cdot 4(0.5), \quad$ yohimbine $=$ $2 \cdot 5$ (1.2), Jonckheere $Z=0.53$, NS; Phase 2 metoprolol $=2 \cdot 0(0 \cdot 9)$, placebo $=2 \cdot 8(0 \cdot 6)$, yohimbine $=3 \cdot 3(0 \cdot 6)$, Jonckheere $Z=1 \cdot 61, P=$ 0.05 ; Phase 3 metoprolol $=1 \cdot 1(0 \cdot 9)$, placebo $=$ $1 \cdot 1(0 \cdot 8)$, yohimbine $=1 \cdot 4(0 \cdot 9)$, Jonckheere $Z=$ $-1 \cdot 58$, NS). Analysis of the total scores on the multiple choice recognition memory test are presented in Fig. 2. The predicted order effect was again confirmed, with metoprolol treated subjects recognizing fewer, and yohimbine treated subjects correctly recognizing more material relative to placebo. Analysis of the recognition memory data by story phase revealed that all three groups recognized more data from the central emotional phase of the story (Fig. 3), and the predicted ordered effect was observed for phases two and three, but was only statistically significant for phase 3 (Jonckheere $Z=$ $1.90, P<0.05)$.

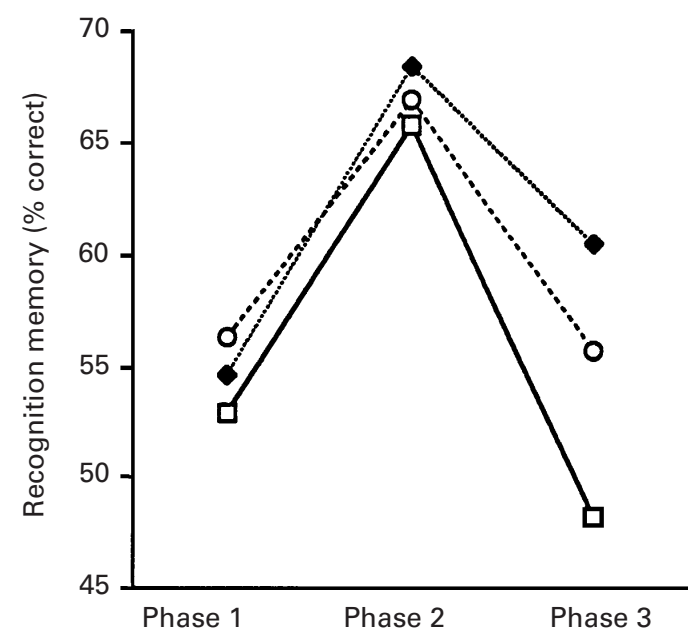

FIG. 3. Heightened memory for the emotive (middle) phase of the story in all three groups $(\square-\square$, metoprolol; $\bullet \cdots \bullet$, yohimbine; $\bigcirc_{--}^{-} \bigcirc$, placebo).

\section{DISCUSSION}

In the present study, random allocation to the three experimental group resulted in good matching in terms of age, sex, intelligence, personality, memory functioning and basal blood pressure and pulse rate. The pharmacological intervention was clearly effective as evidenced by the yohimbine-induced elevations and metoprolol-induced reductions in mean heart rate during the slide presentation. One week later, those subjects who had received yohimbine recalled significantly more, and metoprolol fewer, total number of slides relative to placebo. Thus, stimulation of the noradrenergic system resulted in an enhancement and blockade in a reduction of long-term memory for emotionally valenced material. This effect was particularly marked for the central most emotive section of the presentation. The results of the multiple-choice recognition memory test confirmed the findings for free recall, with yohimbine treated subjects recognizing more material, and metoprolol subjects recognizing less material relative to placebo treated subjects. Analysis of the recognition memory data revealed all groups demonstrated the normative response, i.e. recognizing more of the central emotional phase of the story (Cahill \& McGaugh, 1995). The predicted ordered effect of yohimbine, then placebo followed by metoprolol was evidenced for phase 2 and was statistically significantly for 
phase 3, but not for the initial neutral story phase. These results cannot be explained by differences in emotional reactions of the subjects to the story, as there was no significant difference between the groups on mean arousal ratings immediately following slide presentation, and this suggests a pharmacological effect modulating the encoding of the stimuli.

It is plausible that part of the neural substrate of heightened memory for emotional material involves increased sympathetic arousal (often associated with fear) during the encoding of a traumatic event. Our findings provide new support for the view derived from animal research, that emotional experience activates the noradrenergic system, thus modulating encoding so that incident may be better remembered (Cahill \& McGaugh, 1998). Such a mechanism appears to involve the amygdala (Cahill et al. 1995, 1996) and would have obvious adaptive value in evolutionary terms, so that potentially dangerous situations are clearly remembered and if necessary, avoided in the future. We propose that this heightened noradrenergic tone acts to amplify the signal that is to be remembered. The present results have clear clinical implications. One potential 'down-side' of this modulatory mechanism is that extremely emotional events may be remembered too well by some individuals, leading to intrusive, distressing recollections and flashbacks, the central clinical features of post-traumatic stress disorder (Pitman, 1989).

The effect we observed could possibly have been achieved via the effects of the pharmacological manipulation on peripheral mechanisms. However, both van Stegeren et al. (1998) and O'Carroll et al. (1999) failed to find any effect of peripheral beta blockade on memory for emotional material in man.

Limitations of the present study are that the observed between group effects, while statistically reliable, were small. However, given that we used single trial dosages of yohimbine and metoprolol, with no adjustment for body weight (Peskind et al. 1995) and relatively crude indices of memory, the positive findings for both free recall and recognition are impressive. Given that significant ordered effects were observed for both recall and recognition data, this suggests a relatively robust phenomenon. However, it is extremely unlikely that a single neurotransmitter system is involved in the enhancement of memory for emotional events. Clearly, the hypothalamo-pituitary-adrenal axis is likely to be involved (Yehuda \& Harvey, 1997) as well as a wide variety of other neurotransmitters, including NMDA, GABA and endorphins (O'Brien \& Nutt, 1998). However, the available evidence from experiments using both animals and man strongly supports the view that the noradrenergic system plays an important role (Cahill \& McGaugh, 1998).

We found clear evidence of metoprolol impairing memory for emotional material. Attempts to replicate the original propranolol effect (Cahill et al. 1994) have produced conflicting results (van Stegeren et al. 1998; O'Carroll et al. 1999). This may be because the effect on memory for emotional events is primarily a beta- 1 receptor effect, and propranolol is a non-specific beta-1/beta-2 blocker. We agree with (Cahill et al. (1994) who stated 'Future research using beta blockers with different affinities for beta- 1 and beta- 2 receptors and differing effectiveness in passing the blood-brain barrier should clarify the role of the beta-adrenergic system in regulating long-term memory for emotional experience' (p. 704). The present results of enhanced memory for emotional material also require replication using different pharmacological manipulations. In particular, yohimbine does not produce effects specific to the noradrenergic system (den Boer \& Westenberg, 1993) and further experiments with pharmacological agents with greater specificity for the noradrenergic system are required, as are dose response studies. However, our findings provide support for the hypothesis that increased noradrenergic tone at encoding may be causally implicated in the phenomena of enhanced memory for emotional events in man.

This work was supported by a grant to R. O'C. for the Wellcome Trust ref. no. 048090/Z/96/Z. We would like to thank the subjects who participated in the present study, the staff at the Pharmacy of the Royal Edinburgh Hospital, Sigma Chemical for providing yohimbine, and Norma Brearley for her careful preparation of this manuscript.

\section{REFERENCES}

Cahill, L. \& McGaugh, J. L. (1995). A novel demonstration of enhanced memory associated with emotional arousal. Consciousness and Cognition 4, 410-421. 
Cahill, L. \& McGaugh, J. L. (1998). Mechanisms of emotional arousal and lasting declarative memory. Trends in Neurosciences 21, 294-299.

Cahill, L., Prins, B., Weber, M. \& McGaugh, J. L. (1994). $\beta$ adrenergic activation and memory for emotional events. Nature 371, $702-704$.

Cahill, L., Babinsky, R., Markowitsch, H. J. \& McGaugh, J. L. (1995). The amygdala and emotional memory. Nature 377, 295-296.

Cahill, L., Haier, R. J., Fallon, J., Alkire, M. T., Tang, C., Keator, D., Wu, J. \& McGaugh, J. L. (1996). Amygdala activity a encoding correlated with long-term, free-recall of emotional information. Proceedings of the National Academy of Sciences, USA 93, 8016-8021.

Charney, D. S., Woods, S. W., Goodman, W. K. \& Heninger, G. R (1987). Neurobiological mechanisms of panic anxiety: biochemical and behavioural correlates of yohimbine-induced panic attacks. American Journal of Psychiatry 144, 1030-1036.

Christianson, S. A. (1992). Handbook of Emotion and Memory: Current Research and Theory. Erlbaum: New Jersey.

Coolican, H. (1994). Research Methods and Statistics in Psychology. Hodder \& Stoughton: London.

Delis, D. C., Kramer, J. H., Kaplan, E. \& Ober, B. A. (1987). California Verbal Learning Test - Adult Version Research Manual. The Psychological Corporation: San Antonio, TX

den Boer, J. A. \& Westenberg, H. G. M. (1993). Critical notes on the locus coeruleus hypothesis of panic disorder. Acta Neuropsychiatrica 5, 48-54.

Eysenck, S. B. G., Eysenck, H. J. \& Barrett, P. (1985). A revised version of the psychoticism scale. Personality and Individual Differences 6, 21-29.

Ikeda, M., Mori, E., Hirono, N., Imamura, T., Shimomura, T.,
Ikejiri, Y. \& Yamashita, H. (1998). Amnestic people with Alzheimer's disease who remembered the Kobe earthquake. British Journal of Psychiatry 172, 425-428.

James, W. (1884). What is emotion? Mind 9, 188-205.

LeDoux, J. (1996). The Emotional Brain. Simon \& Schuster Publishers: New York.

Nelson, H. E. \& Willison, J. R. (1991). The Revised National Adult Reading Test - Test Manual. NFER-Nelson: Windsor.

O'Brien, M. \& Nutt, D. (1998). Loss of consciousness and posttraumatic stress disorder. British Journal of Psychiatry $\mathbf{1 7 3}$, $102-104$.

O'Carroll, R. E., Drysdale, E., Cahill, L., Shajahan, P. \& Ebmeier, K. P (1999). Memory for emotional material: a comparison of central versus peripheral beta blockade. Journal of Psychopharmacology 13, 32-39.

Peskind, E. R., Wingerson, D., Murray, S., Pascualy, M., Dobie, D. J., Le Corre, P., Le Verge, R., Veith, R. C. \& Raskind, M. A. (1995). Effects of Alzheimer's disease and normal aging on cerebrospinal fluid norepinephrine responses to yohimbine and clonidine. Archives of General Psychiatry 52, 774-782.

Pitman, R. K. (1989). Post-traumatic stress disorder, hormones, and memory. Biological Psychiatry 26, 221-223.

van Stegeren, A. H., Everaerd, W., Cahill, L., McGaugh, J. L. \& Gooren, L. J. G. (1998). Memory for emotional events: differential effects of centrally versus peripherally acting beta-blocking agents. Psychopharmacology 138, 305-310.

Walker, G. (1991). ABPI Data Sheet Compendium 1991-1992. Datapharm Publications Limited: London.

Yehuda, R. \& Harvey, P. (1997). Relevance of neuroendocrine alterations in PTSD to memory-related impairments of trauma survivors. In Recollections of Trauma. Scientific Evidence and Clinical Practice (ed. J. D. Read and D. S. Lindsay), pp. 221-242. Plenum Press: New York. 